\title{
Human herpes virus type 8 among female-sex workers (a review)
}

\begin{abstract}
The prevalence of Human Herpes Virus type 8 (HHV-8) varies from one region to another and by populations. The modes of transmission and risk factors associated with HHV-8 among heterosexual adults are still not clear. HHV-8 has been associated with high risk sexual behavior. Female-sex workers (FSWs) are a high-risk group and therefore more vulnerable to sexually transmitted infections (STIs) compared to women in the general population. This review aimed at determining the global distribution of HHV-8 among FSWs and its associated risk factors. The secondary aim was to determine transmission and HHV-8 co-infection with Human Immunodeficiency Syndrome (HIV) among FSWs We systematically searched literature in PubMed and Google Scholar. Twenty six studies among FSWs and women engaging in high risk sexual behavior from Africa, Europe, North and South America, Asia and the Mediterranean regions from 1996- 2014 were reviewed. Results were presented in proportions. HHV-8 distribution among FSWs followed patterns of HHV-8 distribution in the general population in the world, that is, high among African and Mediterranean FSWs compared to other regions. The main risk factor associated with HHV-8 was African race. Sexual behavior associated with HHV-8 varied from one study to another. In conclusion, FSWs may not play a vital role in the transmission of HHV-8.
\end{abstract}

Volume 5 Issue 6 - 2017

\author{
Mirriam Mbithe Nzivo,' Raphael M \\ Lwembe, ${ }^{2}$ Eddy O Odari,' Nancy LM \\ Budambula $^{3}$ \\ Jomo Kenyatta University of Agriculture and Technology, Kenya \\ ${ }^{2}$ Center for Virus Research, Kenya Medical Research Institute, \\ Kenya \\ ${ }^{3}$ Department of Biological Sciences, University of Embu, Kenya
}

Correspondence: Mirriam Mbithe Nzivo, Jomo Kenyatta University of Agriculture and Technology, PO. Box 62000, Nairobi, Kenya Email mirriamaquilas@yhoo.com

Received: March 31, 2017| Published: October 03, 2017

Keywords: HHV-8, Female-Sex Workers, Epidemiology, Transmission, Risk factors

Abbreviations: AIDS, Acquired Immune Deficiency Syndrome; BCBL, Body Cavity Based Lymphoma Cell Line; CSWS, Commercial Sex Workers; DNA, Deoxyribonucleic Acid; EIA, Enzyme Immune Assays; ELISA, Enzyme Linked Immunosorbent Assays; FSWS, Female Sex Workers; HBV, Hepatitis B Virus; HCV, Hepatitis C Virus; HHV-8, Human Herpes Virus Type 8; HIV, Human Immunodeficiency Virus; HPV, Human Papilloma Virus; IDUS, Intravenous Drug Users; IFA, Immuno florescence Assay; KSHV, Kaposi's Sarcoma Associated Herpes Virus; KS; Kaposi's Sarcoma; LTR, Long Terminal Repeat; MCD, Multicentric Castleman's Disease; MSMS, Men Having Sex With Men; ORF, Open Reading Frame; PCR, Polymerase Chain Reaction; PEL, Primary Effusion Lymphoma; STDS, Sexually Transmitted Diseases; STIS, Sexually Transmitted Infection; TAT, Trans activator Of Transcription; US, United States

\section{Introduction}

Human herpes virus type 8 also known as Kaposi's sarcoma associated Herpes virus (KSHV) was first identified in 1994, in lesions of patients with Kaposi's sarcoma (KS). ${ }^{1}$ Human herpes virus type $8(\mathrm{HHV}-8)$ is a double stranded DNA virus and the most recently discovered human tumor virus. ${ }^{2}$ It is the underlying cause of all forms of Kaposi's sarcoma including; classic, endemic, AIDSassociated KS and iatrogenic KS. Other diseases associated with the virus are primary effusion lymphoma (PEL) and multicentric castleman's disease (MCD). KS is an AIDS-defining illness and is the second most common malignancy present in HIV-1 infected patients, worldwide. ${ }^{3,4}$ The prevalence of HHV-8 has been reported to be high in high-risks groups such as female sex workers, men having sex with men (MSMs), intravenous drug users (IDU) and HIV patients..$^{5-7}$ The modes of transmission of the virus can be either sexual or non-sexual depending on the group. ${ }^{2}$ There is an inconsistency in reporting about the risk factors associated with the virus by different studies. This review summarizes results of mostly cross-sectional studies among
FSWs, women attending sexually transmitted disease (STD) clinics and those reported to be engaging in high-risk sexual behavior that have contributed to further understanding of HHV-8 distribution and risk factors associated with the virus and also HHV-8 transmission and co-infection with HIV.

\section{Epidemiology of HHV-8}

HHV-8 distribution in the world is not ubiquitous. The epidemiologic patterns of HHV-8 differ by population and geography. The prevalence is high in areas where non-HIV associated forms of KS (classic or endemic forms) have been common like Africa and the Mediterranean. ${ }^{2}$ Sero-epidemiological surveys have reported up to $50 \%$ prevalence in Sub-Saharan Africa in the general population where KS is endemic. In Europe and United States HHV-8 prevalence is low among the general population but significantly high among men having sex with men (MSMs) and in migrants from endemic regions. ${ }^{4,8}$ Prevalence ranges of $20 \%-60 \%$ have been reported among MSMs in United States (US) and Europe. A prevalence of 8.2\% was reported among MSMs in the US. ${ }^{5}$ Casper et al. ${ }^{9}$ reported a prevalence of $23 \%$ among HIV-positive adolescent MSMs. In South America, the prevalence of HHV-8 has been observed to be high among Amerindians across all ages unlike among other populations in the region. In the Mediterranean, the prevalence ranges between $10-40 \%$ and is $0-20 \%$ in most parts of Asia. ${ }^{1,2}$ A study carried out in different countries in the world among women in the general population reported HHV8 prevalence of; In Nigeria $46.02 \%$, Colombia $13.32 \%$, Costa Rica 9.81\%, Argentina $6.40 \%$, Ho Chi Minh City, Vietnam $15.50 \%$, in Hanoi, Vietnam $11.26 \%$, Songkla, Thailand 10\%, Lampang, Thailand $8.63 \%$, Korea $4.93 \%$ and $3.65 \%$ in Spain. ${ }^{10}$

The prevalence of HHV-8 and the incidence of Kaposi's sarcoma in Africa are generally low in Western, Northern and Southern Africa as compared to Eastern and Central Africa. ${ }^{11}$ Equatorial Africa has acquired the name Kaposi's sarcoma belt due to high incidences of $\mathrm{KS}$ even before the HIV pandemic. A study in Uganda within KS belt 
and Zimbabwe and South Africa outside KS belt reported prevalence of $47.1 \%, 24.7 \%$ and $23.1 \%$ respectively. ${ }^{12} \mathrm{HHV}-8$ prevalence in SubSaharan Africa is common in early childhood with the prevalence increasing throughout childhood. HHV-8 seropositivity was also found to be higher in Tanzania within KS belt increasing from 3.7\% among infants to $58.1 \%$ among children aged 3 to 4 years and $89 \%$ among adults $\geq 45$ years. In Nigeria which is outside KS belt, a HHV8 seroprevalence of $22 \%$ and $14 \%$ was reported among men and women in the general population, respectively. ${ }^{13,14}$ General and clinic population- based studies in Uganda reported HHV-8 seroprevalence increased from about $9 \%$ in children 2 years of age to $36 \%$ in children 2 to 8 years age. A HHV- 8 seroprevalence of $26 \%$ was reported among Ethiopian immigrants residing in Israel and 53\% among pregnant women attending antenatal clinic. ${ }^{15}$ A study among sexually active and sexually naïve adults in Uganda reported a HHV-8 prevalence of $56.2 \%$ and 55.4 respectively. ${ }^{6}$ In Kenya, HHV-8 prevalence of $43 \%$ and $44 \%$ were reported among drivers of a trucking company and FSWs, respectively. ${ }^{16}$

\section{Occurrence and Transmission of HHV-8}

The routes of transmission HHV-8 have to yet be clearly defined. A number of studies have reported that HHV-8 can be transmitted during childhood or adulthood via sexual and nonsexual routes. These include, through body fluids such as blood and blood products, saliva, oropharyngeal mucosa secretions, semen and cervico-vaginal secretions and rarely via organ transplantation which represent both vertical and horizontal transmission. ${ }^{2,17,18}$ The modes of transmission of HHV-8 vary depending on the region. In Sub-Saharan Africa, marked differences in the geographic distribution of HHV-8 and KS suggest that environmental cofactors influence HHV-8 transmission, control, and progression to KS. ${ }^{12}$ Sexual transmission has been reported to be the route of transmission of HHV-8 in non-endemic areas, especially among adults. In endemic areas HHV-8 is transmitted through nonsexual routes like via saliva during childhood from mother to child and between sibling. ${ }^{2,4}$ However the risk factors that predispose children to HHV-8 are not yet fully understood.

HHV-8 can be transmitted from mother to child vertically. There have been reports of evidence of HHV-8 in cervical-vaginal secretions of HHV-8 and HIV-1 co-infected women suggesting possible transmission vertically. ${ }^{2}$ Breast feeding has also been reported to transmit HHV-8 from mother to infants ${ }^{19}$ but there is inconsistency in reporting ${ }^{2}$ Saliva has been reported to be the dominant conduit of HHV-8 spread in Africa. HHV-8 is consistently detected in the or pharynx. Studies have reported up to $15-57 \%$ HHV-8 DNA in salivary sample of asymptomatic HHV-8 positive individuals. ${ }^{20}$ A study evaluating shedding rate of HHV-8 DNA among HIV-1 seronegative and seropositive women in Kenya at different oral and genital mucosa sites reported $32 \%, 28 \%, 4 \%, 2.3 \%, 9 \%$ and $18 \%$ in saliva, mouth swabs, cervical, vaginal mucosa, plasma and peripheral blood mononuclear cells respectively. ${ }^{21}$ In Kenya a HHV-8 shedding rate of $23 \%$ in the saliva was reported among $40 \mathrm{HHV}-8$ positive women. Low CD4 count $<500$ among HIV-positive women was associated with high copy number and high shedding rate. ${ }^{20}$ Although transmission of HHV-8 via blood transfusion has been reported, it is uncommon because HHV-8 circulates in B lymphocytes and are scarce in most types of transfusion or lack of viraemia even in HHV-8 infected individuals. ${ }^{22,23}$

Some studies among high-risk groups have reported evidence of sexual transmission. For instance, among men having sex with men (MSMs), HHV-8 has been linked with sexual behavior including number of lifetime partners. ${ }^{7,9}$ In Kenya and Nigeria, studies among female-sex workers (FSWs) have reported evidence of heterosexual transmission with risk of infection increasing with multiple number of sexual partners, history of sexually transmitted infections (STIs), age and HIV seropositivity. ${ }^{16,24}$ However, the possibility of sexual transmission among heterosexual individuals remains unclear. ${ }^{5-7}$

\section{Role of sex workers in transmission of HHV-8}

Worldwide, sex workers are disproportionately affected by the HIV pandemic. A review of HIV burden in FSWs in 50 lowincome and middle- income countries reported an overall HIV prevalence of $11.8 \%$, with a pooled odds of HIV infection of 13.5 compared with the general population of women of reproductive age. ${ }^{25} \mathrm{FSWs}$ therefore, represent heterosexual transmission and are an important group in understanding sexual behavior involved in HHV8 transmission. Commercial sex has been reported as a risk factor of HHV-8 especially among women. ${ }^{6,13}$ The prevalence of HHV-8 in women in general low-risk population has in some studies been reported to be low as compared to that of FSW. ${ }^{13,23,24}$ However, there is a contradiction in reporting by different studies on the role of sex workers in transmission of HHV-8. For instance studies in Honduras, US, Kenya, Uganda and Nigeria reported that sex work plays a major role in transmission of HHV-8..$^{6,16,22,26}$ However, studies in Djibouti, South Africa and China, reported sex work to be not-significant in the transmission of HHV-8. ${ }^{27-29}$ The lack of association of HHV-8 with sexual behavior by several studies casts doubt on possibility of HHV-8 transmission sexually. The major challenge in determining role of sexual transmission of HHV-8 is that both kissing and salivary exchange are common during heterosexual and homosexual contact. This makes it harder to determine whether the transmission was sexual or oral through saliva. FSWs are also a potential threat to other lowrisk population and therefore, understanding modes of transmission and risk factors associated with HHV-8 is essential for development of prevention strategies of HHV-8 transmission and diseases associated with this virus.

\section{HHV-8 infection among Female Sex Workers}

The prevalence of HHV-8 among FSWs varies considerably from one region to another one like the prevalence of the general population. In the late 1990s, a study among HIV-positive prostitutes in Honduras reported a HHV-8 prevalence of $36 \%$ among FSWs compared to $9.9 \%$ among non-FSWs. ${ }^{26}$ In Cameroon, Central Africa, HHV-8 prevalence of $51.3 \%$ among FSWs and $36.6 \%$ in HIV pregnant women was reported..$^{30}$ In Eritrea, a low HHV-8 seroprevalence of $8 \%$ was documented among FSWs as compared to 5\% prevalence among pregnant women and $26 \%$ among members of an isolated Rashaida tribe. ${ }^{31}$ At the beginning of 21 st century, a HHV- 8 prevalence of $42 \%$ and $11.5 \%$ was reported amongst prostitutes and general population women in Western Sicily, respectively. ${ }^{32}$ In Spain, HHV-8 prevalence of $16 \%$ and $8 \%$ was documented among prostitutes and general population women, respectively. In the same study, Spanish prostitutes had a lower HHV-8 prevalence (12\%) compared to foreign prostitutes $(17.3 \%) .{ }^{33}$ Eltom et al. ${ }^{13}$ reported a HHV-8 prevalence of $31 \%$ among Commercial sex workers (CSWs) as compared to $19 \%$ among lowrisk men and women in the general population. In Djibouti female prostitutes working in the streets recorded HHV-8 prevalence of $26 \%$ while those working in luxury bars $20 \% .{ }^{27} \mathrm{HHV}-8$ prevalence of $10 \%$, $10 \%$ and $25 \%$ in general population women, HIV-positive women and prostitutes respectively was reported in Spain. ${ }^{34}$ A cohort study among HIV-1 seronegative prostitutes in Mombasa, Kenya reported a HHV-8 prevalence of $44 \% .{ }^{16}$ In Italy, a study among FSWs reported a HHV-8 prevalence of $32.7 \%$. In the same study, when HHV-8 prevalence was determined, based on region, foreign prostitutes most of whom were 
from Nigeria had a HHV-8 prevalence of $42.9 \%$ compared to $10.3 \%$ among those of Western Europe origin. ${ }^{35}$ Caterino de Araujo et al. ${ }^{36}$ in Imbituba, Santa Catarina Brazil reported a HHV-8 prevalence of $6.7 \%$ among FSWs. In South Africa, FSWs, miners and male and female residents of a township recorded a prevalence of HHV-8 of 50.5\%, $48.4 \%, 47.5 \%$ and $46 \%$, respectively. ${ }^{28}$

Studies carried out in the past five years also show variation in different geographical regions; a study in China among FSWs, Intravenous drug users (IDUs) and a healthy population among others reported HHV-8 prevalence of $16.7 \%, 58.7 \%$ and $13.1 \%$, respectively. ${ }^{23} \mathrm{HHV}-8$ prevalence was reported as $45 \%$ among FSWs in Jos, Nigeria. ${ }^{24}$ In Shanghai, China, a HHV-8 prevalence of $10 \%$ was recorded among FSWs; while for women in the general population was $11 \% .^{29}$ This was lower than HHV-8 prevalence of $22.2 \%, 31.2 \%$ and $32.7 \%$ reported among immune compromised patients, IDUs and homosexual men respectively in the same country. ${ }^{23}$ The different studies on HHV-8 among FSWs are summarized in (Table 1). There is no gold standard test for HHV-8 making it difficult to compare different studies as various assays perform differently. Researchers have used serological assays such as Enzyme Linked Immunosorbent assay (ELISA) and Immunofluorescence assays (IFAs) over the years as HHV-8 DNA is not always detectable in positive individuals. The prevalence varied from one region to another. The number of studies carried out in each continent among FSWs and the maximum prevalence observed are summarized in (Table 2) Majority of the studies reviewed were from Africa and recorded the highest prevalence $(51.3 \%)$ followed by the Mediterranean (42\%). Studies from Asia and N. America recorded the lowest prevalence $16.7 \%$ and $17.3 \%$, respectively. In general, studies on HHV-8 among FSWs are limited.

Table I Report summarizing prevalence of HHV-8 among FSWs between 1998 and 2017 and the type of assays used

\begin{tabular}{llll}
\hline Country & Prevalence (\%) & Assay & Author(S) \\
\hline Cameroon & $5 I .3$ & Immunoperoxidase test & Betsetti et al. [30] \\
Honduras & 36 & IFA, radioimmunoprecipitation & Sosa et al. [26] \\
Eritrea & 8 & IFA & Enbom et al. [3I] \\
Sicily & 42 & Indirect IFA & Perna et al. [32] \\
Spain & 16 & Latent IFA, ELISA (K8.I) and PCR & De Sanjose et al. [33] \\
Djibouti & 26 and 20 & Indirect IFA (BC-3 cell line) & Marcelin et al. [27] \\
Nigeria & 31 & EIA (K8.I) & Eltom et al. [13] \\
Spain & 25 & PCR & Santon et al. [34] \\
Kenya & 44 & ELISA (whole-virus lysate) & Lavrey et al. [16] \\
USA & 17.3 & serological assay (ORF 65 and K8.I) & Stover et al. [37] \\
Italy & 32.7 & IFA (BCBL-I cell line) & Smacchia et al. [35] \\
Brazil & 6.7 & In-house IFA \&Western blot & Caterino de Araujo et al. [36] \\
South Africa & 50.5 & ELISA (K 8.I and ORF 73) & Malope et al. [28] \\
China & 16.7 & ELISA (ORF 65) & Ju et al. [23] \\
Nigeria & 45 & ELISA & Zakari et al. [24] \\
China & 10 & IFA (lytic or latent antigen) & Zhang et al. [29]
\end{tabular}

Table 2 Summary of number of studies among FSWs undertaken in each continent and the recorded highest prevalence

\begin{tabular}{lll}
\hline Continent & No. of Studies & Maximum (\%) Prevalence \\
\hline Africa & 7 & 51.3 \\
N.America & 1 & 17.3 \\
S.America & 2 & 36 \\
Europe & 3 & 32.7 \\
Mediterranean & $\mathrm{I}$ & 42 \\
Asia & 2 & 16.7 \\
\hline
\end{tabular}

\section{HHV-8 and HIV co-infection among FSWS}

HHV-8 has been reported to be higher among HIV-positive patients compared to HIV negative individuals. ${ }^{7,17,24}$ HIV-positive adults have an up to 20000 fold increase of developing KS than the general population and 300 fold higher than in persons with any type of immuno suppression. ${ }^{38} \mathrm{HHV}-8 / \mathrm{HIV}$ co-infection has been reported by a number of studies. In Burkina Faso a study among pregnant women reported a HHV-8/HIV co-infection prevalence of $12.5 \% .^{39}$ The prevalence of HHV-8/HIV co-infection among Zimbabwean men was reported as $6.5 \% .^{40} \mathrm{FSWs}$ in Jos, Nigeria and Imbituba, Brazil recorded a prevalence of HHV-8/HIV co-infection of $20 \%$ and $16.7 \%$, respectively. ${ }^{24,36}$ There is however conflicting information concerning HHV-8/HIV association which could be attributed to diverse range of populations studied with different transmission routes. ${ }^{7}$ In Nigeria, the Seroprevalence of HHV-8 infection was reported as $62 \%$ in HIV1-positive patients and $25.9 \%$ in HIV-negative adults. ${ }^{17}$ In Zambia, HHV-8 positive women were reported to be more likely co-infected with HIV-1 than those who were HHV-8 negative $34 \%$ versus $26 \%$, respectively. ${ }^{41}$ Dramatically high HHV-8 Seroprevalence of $80 \%$ and $81 \%$ were observed among HIV negative and HIV-positive individuals in Cameroon respectively indicating, HHV-8 is high in the general population in the country. ${ }^{42}$

Sex workers and women engaging in high-risk sexual behavior are likely to be co-infected with HHV-8 and HIV than the general population. Honduras HIV-positive FSWs recorded a prevalence of $36 \%$ compared to $9.9 \%$ among HIV negative non-FSWs. ${ }^{26}$ In Western Sicily, a HHV- 8 prevalence of $42 \%$ was reported among HIV-positive female prostitutes compared to $30 \%$ among HIV negative ones. ${ }^{32}$ Greenblatt et al. ${ }^{43}$ reported a prevalence of HHV- 8 of $15 \%$ among HIVpositive and $6.3 \%$ among HIV negative high-risk women. In Spain, HHV-8 Seroprevalence was also reported to be higher among HIVpositive FSWs with a pooled odds ratio of 4.1 compared to the HIV negative ones. ${ }^{35}$ Zakari et al. ${ }^{24}$ also observed the prevalence of HHV8 to be higher among HIV-positive FSWs than HIV negative ones 
$(\mathrm{p}<0.05)$. However, a large study in South Africa among sex workers, miners and residents of a township showed there was no significant difference in the prevalence of HHV-8 among HIV-positive and HIV negative individuals reporting $48.5 \%$ and $46.8 \%$ respectively. ${ }^{28}$ In Uganda there was no significant association of infection with the two viruses $(p=0.660)$ among sexually active and sexually naïve adults. ${ }^{6}$ A study in the US among several cohorts including women attending STD clinic reported a prevalence of 9\%. HIV-positive heterosexuals reported $0 \%$ while HIV negative heterosexuals recorded $7 \% .{ }^{44}$

There is scientific evidence however that HHV-8 and HIV interact regulating infection with each other and in human carcinogenesis. The level of interaction is largely determined by the route of transmission, risk factors involved and type of cell infected. Molecular interactions between the viruses also play an important role in progression of diseases caused by the two viruses ${ }^{45}$ HHV- 8 replication can be induced by cytokines such as interlukins 1 , interferon- $F$, oncostatin $\mathrm{M}$, hepatocyte growth factors/ Scatter factors which are produced due to HIV infected cells. HIV infection stimulates replication of HHV-8 in co-infected and adjacent cells. ${ }^{38}$ Trans- activator of transcription (Tat) protein of HIV enhances the lytic cycle replication of HHV-8, proliferation and anti-apoptosis of infected spindle cells and also aids in tumor genesis of endothelial cells via activation of vascular endothelial growth factor. ${ }^{38,46,47}$ Conversely, HHV-8 promotes progression of HIV to AIDS. HHV-8 promotes transendothelial spread of HIV by inducing HIV replication as well as promoting infection of new HIV target cells. ${ }^{17} \mathrm{HHV}-8$ latency associated nuclear antigen activates LTR of HIV and stimulates expression of HIV P24. In addition, immediate early gene ORF 50 (the major trans activator of HHV-8 lytic cycle) acts synergistically with HIV-1 Tat protein at post transcriptional level enhancing HIV-1 LTR transactivation. This leads to potent stimulation of HIV-1 gene expression. It also enhances HIV replication at $\mathrm{T}$ and $\mathrm{B}$ cells and induces susceptibility and permissiveness in non-susceptible cells. ${ }^{45-47}$

\section{Risk factors associated with HHV-8 infection}

There are several risk factors that have been reported to influence transmission of HHV-8. They range from socioeconomic factors such as low income, poor hygiene, low level of education, and increased number of children born and age to environmental and geographical characteristics such as volcanic soils, plant, itchy insect bites and African descent among others. ${ }^{5,6,10,14}$ Other factors are associated with sexual behavior such as; marital status, frequent alcohol drinking, multiple sex partners, engaging in sex at an early age, HIV, HPV, HBV, HSV-2 infections, history of STIs such as gonorrhea, syphilis, Chlamydia trachoma is, Condyloma acuminate, occasional condom use, increased duration as a sex worker, among others..$^{13,24,29,36}$ Risk factors that influence transmission of HHV-8 among FSWs and women believed to have high-risk sexual behavior are summarized in (Table 2). HBV- Hepatitis B virus, Hepatitis C virus, HIV- Human Immunodeficiency virus, HPV- Human papilloma virus, IDUIntravenous drug use, STDs- Sexually transmitted diseases. Risk factors associated with HHV-8 infection among female sex workers varied from one study to another (Table 3 ).

(Table 4) summarizes the total number of studies that evaluated a certain risk factor, the number of studies that observed significant association of the risk factor with HHV-8 and number of studies that did not observe significant association. African race or country of origin especially African countries was to significantly associate with HHV-8 by all 7 studies that tested it. The other factors that stood out were HIV and history of STDs. Ten (62\%) out of the 16 studies that evaluated HIV as risk factor reported significant association. Seven (58\%) out 12 studies reported significant association with STDs while three $(60 \%)$ out of five studies that tested IDU reported significant association. Age was evaluated by many studies but only $6(40 \%)$ out of the 16 reported significant associations with HHV-8. Having multiple sexual partner was evaluated by 14 studies but only $2(15 \%)$ reported significant association. The other factors; HSV-2, HBV, duration of sex work, age at first sexual intercourse, were reported by most studies evaluating them as non-significant. The number of studies that reported significant and non-significant association of HHV-8 with various risk factors are reported in (Figure 1). Majority of studies reported non-significant association of HHV-8 with most of the risk factors the review evaluated.

Table 3 Risk factors associated with HHV-8 among female sex workers and women engaging in high risk sexual behavior

\begin{tabular}{|c|c|c|c|}
\hline Type of Population & Country & Risk Factors & Author(S) \\
\hline High risk sexual behavior & USA & Age, HIV, Syphilis & Kedes et al. [44] \\
\hline Prostitutes & Cameroon & Age, HIV seropositivity & Betsetti et al. [30] \\
\hline Commercial sex workers & Honduras & $\begin{array}{l}\text { HIV, History of STI, engaging in early sex intercourse }>16 \text {, multiple sex } \\
\text { partners, abnormal pap smear }\end{array}$ & Sosa et al. [26] \\
\hline Prostitutes & Eritrea & None of the risk factors tested were significant (HIV and HSV-2) & Enbom et al. [3I] \\
\hline STD clinic attendees & \multicolumn{2}{|c|}{ United Kingdom African descent } & Smith et al. [48] \\
\hline High risk sexual behavior & Sweden & $\begin{array}{l}\text { High number of sexual partners(>10), history of STDs, Chlamydia } \\
\text { trachomatis, Condyloma acuminata }\end{array}$ & Tedeschi et al. [49] \\
\hline Female prostitutes & Sicily & Age, HIV, STI & Perna et al. [32] \\
\hline $\begin{array}{l}\text { Women with high risk sexual } \\
\text { behavior and drug use }\end{array}$ & USA & $\begin{array}{l}\text { African descent, lower level of education, infection with syphilis, HIV, } \\
\text { HBV, HCV, IDU, commercial sex }\end{array}$ & Cannon et al. [22] \\
\hline High risk sexual behavior & USA & $\begin{array}{l}\text { HIV, older age, syphilis, African descent, crack, cocaine, heroin, IDU male } \\
\text { sex partner }\end{array}$ & Greenblatt et al. [43] \\
\hline STD clinic attendees & USA & $\begin{array}{l}\text { None of the risk factors tested were significant among female } \\
\text { heterosexuals }\end{array}$ & Gambus et al. [50] \\
\hline Female sex workers & Spain & $\begin{array}{l}\text { Early age at first sex intercourse, presence of HPV DNA, country of } \\
\text { origin (S.America) }\end{array}$ & De Sanjose et al. [33] \\
\hline Prostitutes & Djibouti & None of the risk factors tested were significant & Marcelin et al. [27] \\
\hline Female sex workers & Nigeria & Increasing age, history of STDs, multiple sex partners & Etom et al. [13] \\
\hline STD clinic attendees & France & Country of origin, HSV-2 infection & Janier et al. [5I] \\
\hline Prostitutes & Spain & Geographic origin & Santon et al. [34] \\
\hline
\end{tabular}


Table Continued...

\begin{tabular}{|c|c|c|c|}
\hline Type of Population & Country & Risk Factors & Author(S) \\
\hline Prostitutes & Kenya & $\begin{array}{l}\text { Age, years of education, work place, hormonal contraception, IUD, } \\
\text { alcohol consumption, syphilis, gonorrhea }\end{array}$ & Lavrey et al. [16] \\
\hline High risk sexual behavior & USA & HIV, African race and age group $36-45$, IDU & Stover et al. [37] \\
\hline Prostitutes & Italy & HIV, country of origin & Smacchia et al. [35] \\
\hline High risk sexual behavior & USA & History of gonorrhea, having sex with women, African American race & Casper et al. [9] \\
\hline Female sex workers & Brazil & Age, African descent, History of STDs & Caterino de Araujo et al. [36] \\
\hline High risk sexual behavior & USA & Multiple number of sexual partners $(>10)$ & Giuliani et al. [52] \\
\hline Sex workers & South Africa & frequent alcohol drinking, marriage & Malope et al. [28] \\
\hline Sexually active VS naïve adults & Uganda & Age, two vs. one marital union, increased number of children, CSW & Shebl et al. [6] \\
\hline Female sex workers & China & Intravenous drug use & Ju et al. [23] \\
\hline Commercial sex workers & Nigeria & HIV, History of STI & Zakari et al. [24] \\
\hline Female sex workers & China & None of the risk factors tested were significant & Zhang et al. [29] \\
\hline
\end{tabular}

Table 4 Percent number of studies reporting significant and non-significant association of different risk factors associated with HHV-8

\begin{tabular}{llll}
\hline \multirow{2}{*}{ Risk Factor } & Total Studies & Significant Association & Non-Significant Association \\
\cline { 2 - 4 } Age & $\mathbf{N}=\mathbf{2 6}$ & $\mathbf{N}(\%)$ & $\mathbf{N}(\%)$ \\
HIV & 16 & $6(37.5)$ & $10(62.5)$ \\
Syphilis & 16 & $10(62.5)$ & $6(37.5)$ \\
Gonorrhea & $1 \mathrm{C}$ & $4(36.4)$ & $7(63.6)$ \\
HSV-2 & 8 & $2(25)$ & $6(75)$ \\
HBV & 8 & $1(12.5)$ & $6(87.5)$ \\
Chlamydia & 5 & $1(20)$ & $4(80)$ \\
STD history & 5 & $1(20)$ & $4(80)$ \\
IDU & 12 & $7(58.3)$ & $5(41.7)$ \\
Multiple partners & 4 & $2(50)$ & $2(50)$ \\
Condom use & 14 & $2(14.3)$ & $12(85.7)$ \\
Age at sex debut & 6 & $2(33.3)$ & $45(66.7)$ \\
Duration of prostitution & 7 & $1(14.3)$ & $6(85.7)$ \\
African race/origin & 5 & $1(20)$ & $5(80)$ \\
\hline
\end{tabular}

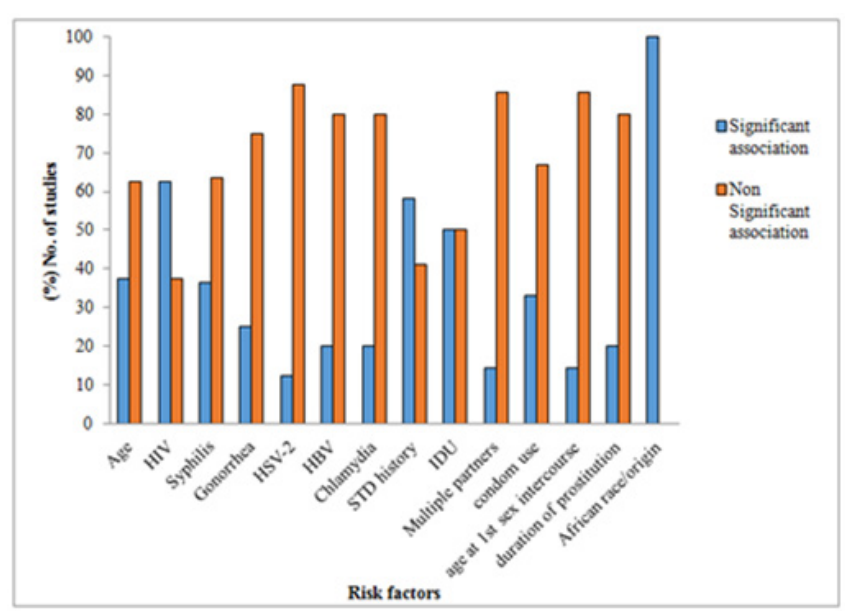

Figure I Risk factors as reported by the reviewed studies.

\section{Conclusion}

The prevalence of HHV-8 among FSWs mirrors HHV-8 distribution in different region with high prevalence reported among FSWs from African countries. HHV-8 prevalence was high among FSWs in Africa and the Mediterranean ${ }^{16,24,28,32}$ as compared to United States (US) and Asia. ${ }^{29,36}$ The low prevalence of HHV-8 among FSWs in some region could be due to the fact that HHV-8 prevalence in the general population in such regions is low. ${ }^{2,29,36}$ It is difficult to conclude whether HHV-8 prevalence had increased or decreased over years as most studies did not report follow up data of the research group over time. There was evidence that heterosexual transmission of HHV-8 occurred in some studies. ${ }^{16,24,26}$ However in other countries, sexual transmission of HHV-8 among heterosexuals was insignificant route of HHV-8 transmission. ${ }^{5,6,29}$ The sexual behaviors surrounding heterosexual transmission are not clear. HHV-8 did not in some studies present with characteristics of a STI. The lack of association of HHV-8 with high-risk sexual behavior and markers of sexual promiscuity such as HIV, STDs like syphilis and gonorrhea, HSV2, HBV infection among FSWs and women engaging in high-risk sexual behavior generates controversy. ${ }^{5,6,28,29,31}$ Significant association of HHV-8 with STDs reported in some studies could be a marker of promiscuity rather than a co-factor for HHV-8 infection. A recent systemic analysis that included studies from 32 countries around the world, reported HHV-8 was weakly associated with HIV among adults who engage in low-risk and high-risk behavior with pooled odds of 1.42 and 1.66 as compared to MSMs and hemophilia patients (OR 3.95 and 3.11), respectively. ${ }^{7}$ The fact that distribution of HHV-8 followed that of general population in different regions and its lack of association with markers of sexual promiscuity indicate that FSWs therefore may not play a major role in HHV-8 transmission. Factors correlated to geographical characteristics could play major role in transmission of HHV-8.

\section{Limitations and recommendations}

Most of the studies among FSWs were cross sectional studies hence were unable to determine risk behaviors at time of sero conversion. Only found a few studies compared prevalence of HHV8 among FSWs and general population women. Majority studies did not determine HHV-8 subtypes among FSWs hence difficult to 
determine their role in spreading of HHV-8 subtypes from one region to another. Generally, there are limited studies investigating HHV8 transmission and prevalence among FSWs. In order to determine modes of transmission and sexual behaviors associated with HHV-8, large prospective cohort studies among HHV-8 negative FSWs need to be carried out. There is also a need for a gold standard test for HHV8 to ensure uniformity in order to compare results confidently from different regions and populations.

\section{Acknowledgments}

None.

\section{Conflicts of interest}

None.

\section{References}

1. Leao JC, de Faria AB, Fonseca DD, et al. Intrahost genetic variability of human herpes virus-8. J Med Virol. 2013;85(4):636-645.

2. Minhas V, Wood C. Epidemiology and transmission of Kaposi's sarcoma-associated herpesvirus. Viruses. 2014;6(11):4178-4194.

3. La Ferla L, Pinzone MR, Nunnari G, et al. Kaposi's sarcoma in HIVpositive patients: the state of art in the HAART-era. European review for medical and pharmacological sciences. 2013;17(17):2354-2365.

4. Rohner E, Wyss N, Trelle S, et al. HHV-8 seroprevalence: a global view. Systematic reviews. 2014;3:11

5. Engels EA, Atkinson JO, Graubard BI, et al. Risk factors for human herpesvirus 8 infection among adults in the United States and evidence for sexual transmission. J Infect Dis. 2007;196:199-207.

6. Shebl FM, Dollard SC, Pfeiffer RM, et al. Human herpesvirus 8 seropositivity among sexually active adults in Uganda. PLoS One. 2011;6(6):e21286.

7. Rohner E, Wyss N, Heg Z, et al. HIV and human herpesvirus 8 coinfection across the globe: Systematic review and meta-analysis. International journal of cancer. 2016;138(1):45-54.

8. Martin JN. Kaposi sarcoma-associated herpesvirus/human herpesvirus 8 and Kaposi sarcoma. Advances in dental research. 2011;23(1):76-78

9. Casper C, Meier AS, Wald A, et al. Human herpesvirus 8 infection among adolescents in the REACH cohort. Archives of pediatrics \& adolescent medicine. 2006;160(9):937-942.

10. De Sanjose S, Mbisa G, Perez Alvarez S, et al. Geographic variation in the prevalence of Kaposi sarcoma-associated herpesvirus and risk factors for transmission. J Infect Dis. 2009;199(10):1449-1456.

11. Biryahwaho B, Dollard SC, Pfeiffer RM, et al. Gender and geographic patterns of human herpesvirus 8 infection in a nationallyrepresentative population-based sample in Uganda. $J$ Infect Dis. 2010;202(9):1347-1353.

12. Dollard SC, Butler LM, Jones AM, et al. Substantial regional differences in human herpesvirus 8 seroprevalence in sub-Saharan Africa: insights on the origin of the "Kaposi's sarcoma belt". International journal of cancer. 2010;127(10):2395-2401.

13. Eltom MA, Mbulaiteye SM, Dada AJ, et al. Transmission of human herpesvirus 8 by sexual activity among adults in Lagos, Nigeria. Aids. 2002;16(18):2473-2478.

14. Mbulaiteye SM, Pfeiffer RM, Whitby D, et al. Human herpesvirus 8 infection within families in rural Tanzania. $J$ Infect Dis. 2003;187(11):1780-1785.

15. Margalith M, Chatlynne LG, Fuchs E, et al. Human herpesvirus 8 infection among various population groups in southern Israel. J Acquir Immune Defic Syndr. 2003;34:500-505.
16. Lavreys L, Chohan B, Ashley R, et al. Human herpesvirus 8: seroprevalence and correlates in prostitutes in Mombasa, Kenya. J Infect Dis. 2003;187(3):359-363.

17. Ogoina D, Onyemelukwe G, Musa BO, et al. Seroprevalence and determinants of human herpes virus 8 infection in adult Nigerians with and without HIV-1 infection. African health sciences. 2011;11(2):158-162.

18. Zhang T, Liu Y, Zhang Y, et al. Seroprevalence of human herpesvirus 8 and hepatitis $\mathrm{C}$ virus among drug users in Shanghai, China. Viruses. 2014;6(6):2519-2530.

19. Dedicoat M, Newton R, Alkharsah KR, et al. Mother-to-child transmission of human herpesvirus-8 in South Africa. $J$ Infect Dis. 2004;190(6):1068-1075.

20. Phipps W, Saracino M, Selke S, et al. Oral HHV-8 replication among women in Mombasa, Kenya. J Med Virol. 2014;86(10):1759-1765.

21. Taylor MM, Chohan B, Lavreys L, et al. Shedding of human herpesvirus 8 in oral and genital secretions from HIV-1-seropositive and seronegative Kenyan women. J Infect Dis. 2004;190:484-488.

22. Cannon MJ, Dollard SC, Smith DK, et al. Blood-borne and sexual transmission of human herpesvirus 8 in women with or at risk for human immunodeficiency virus infection. The New England journal of medicine. 2001;344(1):637-643.

23. Ju HZ, Zhu B, Wang YJ, et al. Regional prevalence and transmission route of Kaposi's sarcoma-associated herpes virus in Zhejiang, China. Chinese medical journal. 2012;125(4):710-712.

24. Zakari H, Nimzing L, Agabi YA, et al. Seroprevalence of Human Herpes Virus 8 (HHV8) Infection among Commercial Sex Workers in Jos. Bayero Journal of Pure and Applied Sciences. 2012;5(1):159-162.

25. Shannon K, Strathdee SA, Goldenberg SM, et al. Global epidemiology of HIV among female sex workers: influence of structural determinants. The Lancet. 2015;385(3):55-71.

26. Sosa C, Klaskala W, Chandran B, et al. Human herpesvirus 8 as a potential sexually transmitted agent in Honduras. J Infect Dis. 1998;178(2):547-551.

27. Marcelin AG, Grandadam M, Flandre P, Nicand E, Milliancourt C, et al. Kaposi's sarcoma herpesvirus and HIV-1 seroprevalences in prostitutes in Djibouti. J Med Virol. 2002;68(2):164-167.

28. Malope BI, Macphail P, Mbisa G, et al. No evidence of sexual transmission of Kaposi's sarcoma herpes virus in a heterosexual South African population. AIDS. 2008;22(4):519-526.

29. Zhang T, Yang Y, Yu F, et al. Kaposi`s sarcoma associated herpesvirus infection among female sex workers and general population women in Shanghai, China: a cross-sectional study. BMC Infect Dis. 2014;14:58.

30. Bestetti G, Renon G, Mauclere P, et al. High seroprevalence of human herpesvirus-8 in pregnant women and prostitutes from Cameroon. AIDS. 1998;12:541-543.

31. Enbom M, Tolfvenstam T, Ghebrekidan H, et al. Seroprevalence of human herpes virus 8 in different Eritrean population groups. $J$ Clin Virol. 1999;14(3):167-172.

32. Perna AM, Bonura F, Vitale F, et al. Antibodies to human herpes virus type 8 (HHV8) in general population and in individuals at risk for sexually transmitted diseases in Western Sicily. International journal of epidemiology. 2000;29(1):175-179.

33. De Sanjose S, Marshall V, Sola J, et al. Prevalence of Kaposi's sarcoma-associated herpesvirus infection in sex workers and women from the general population in Spain. International journal of cancer. 2002;98(1):155-158.

34. Santon RA, De San Jose S, Gomez SE, et al. Human herpesvirus-8 detection in Kaposi's sarcoma, multiple myeloma, and lymphoproliferative syndromes occurring in immunocompetent and immunocompromised patients. Medicina clinica. 2002;119(7):241-244. 
35. Smacchia C, Parolin A, Piselli P, et al. Infection with Human Herpesvirus Type- 8 among Foreign Female Sex Workers in Italy. Infection. 2004;32(5):303-305.

36. Caterino de Araujo A, Santos Fortuna E, Magri MC, et al. Latent human herpesvirus - 8 (HHV-8) infection in female commercial sex workers from Imbituba, Santa Catarina, Brazil. The Brazilian journal of infectious diseases. 2007;11(1):9-11.

37. Stover CT, Smith DK, Schmid DS, et al. Prevalence of and risk factors for viral infections among human immunodeficiency virus (HIV)-infected and high-risk HIV-uninfected women. J Infect Dis. 2003;187(9):1388-1396.

38. da Silva SR, de Oliveira DE. HIV, EBV and KSHV: viral cooperation in the pathogenesis of human malignancies. Cancer letters. 2011;305(2):175-185.

39. Ilboudo D, Karou D, Nadembega WN, et al. Prevalence of human herpes virus-8 and hepatitis B virus among HIV seropositive pregnant women enrolled in the Mother-to-Child HIV Transmission Prevention Program at Saint Camille Medical Centre in Burkina Faso. Pakistan journal of biological sciences. 2007;10(17):2831-2837.

40. Campbell TB, Borok M, Ndemera B, et al. Lack of evidence for frequent heterosexual transmission of human herpesvirus 8 in Zimbabwe. Clinical infectious diseases: an official publication of the Infectious Diseases Society of America. 2009;48(11):1601-1608.

41. Klaskala W, Brayfield BP, Kankasa C, et al. Epidemiological characteristics of human herpesvirus-8 infection in a large population of antenatal women in Zambia. J Med Virol. 2005;75(1):93-100.

42. Stolka K, Ndom P, Hemingway Foday J, et al. Risk factors for Kaposi's sarcoma among HIV-positive individuals in a case control study in Cameroon. Cancer Epidemiol. 2014;38(2):137-143.

43. Greenblatt RM, Jacobson LP, Levine AM, et al. Human herpesvirus 8 infection and Kaposi's sarcoma among human immunodeficiency virusinfected and -uninfected women. J Infect Dis. 2001;183:1130-1134.
44. Kedes DH, Operskalski E, Busch M, et al. The seroepidemiology of human herpesvirus 8 (Kaposi's sarcoma-associated herpesvirus): distribution of infection in KS risk groups and evidence for sexual transmission. Nat Med. 1996;2(8):918-924.

45. Tugizov SM, Webster Cyriaque JY, Syrianen S, et al. Mechanisms of viral infections associated with HIV: workshop 2B. Advances in dental research. 2011;23(1):130-136.

46. Caselli E, Galvan M, Cassai E, et al. Human herpesvirus 8 enhances human immunodeficiency virus replication in acutely infected cells and induces reactivation in latently infected cells. Blood. 2005;106(8):2790-2797.

47. Pyakurel P, Pak F, Mwakigonja AR, et al. KSHV/HHV-8 and HIV infection in Kaposi's sarcoma development. Infectious agents and cancer. 2007:2:1-4.

48. Smith, N. A. Sabin CA, Gopal R, et al. Serologic evidence of human herpesvirus 8 transmission by homosexual but not heterosexual sex. $J$ Infect Dis. 1999;180(3):600-606.

49. Tedeschi R, Caggiari L, Silins I, et al. Seropositivity to human herpesvirus 8 in relation to sexual history and risk of sexually transmitted infections among women. International journal of cancer. 2000;87(2):232-235.

50. Gambus G, Bourboulia D, Esteve A, et al. Prevalence and distribution of HHV-8 in different subpopulations, with and without HIV infection, in Spain. AIDS. 2001;15(9):1167-1174.

51. Janier M, Agbalika F, de La Salmoniere P, et al. Human herpesvirus 8 seroprevalence in an STD clinic in Paris: a study of 512 patients. Sex Transm Dis. 2002;29(11):698-702.

52. Giuliani M, Cordiali Fei P, Castilletti C, et al. Incidence of human herpesvirus 8 (HHV-8) infection among HIV-uninfected individuals at high risk for sexually transmitted infections. BMC Infect Dis. 2007;7:143. 